\title{
Colaboración, diálogo y reflexión: Una mirada hacia la emergencia de los saberes pedagógicos del siglo XXI
}

\author{
Collaboration, dialogue and reflection: A vision toward the emergence \\ of pedagogical knowledge in the 21st century \\ * Evelyn Zagal Valenzuela
}

Zagal, E. (2021). Colaboración, diálogo y reflexión: Una mirada hacia la emergencia de los saberes pedagógicos del siglo XXI. Revista Convergencia Educativa, (10), diciembre, 7-22. https://doi.org/10.29035/rce.10.7

[Recibido: 07 septiembre, 2021 / Aceptado: 28 octubre, 2021]

\begin{abstract}
RESUMEN
El presente ensayo reflexivo tiene como objetivo reflexionar acerca de los procesos implicados en la acción pedagógica y sostiene la tesis que la emergencia de los saberes pedagógicos necesarios para el siglo XXI, será propiciada, a partir de interacciones dialógicas-participativas, prácticas reflexivas-colaborativas y situadas que realice el profesorado dentro de sus propios contextos educativos. Los procesos vinculados al ámbito pedagógico deben hacer un esfuerzo por lograr avanzar hacia una propuesta reformadora, que invite a construir un camino hacia la transformación. Por ello, resulta imperioso que el profesorado sea capaz de comprender, aprehender y reflexionar acerca de los dilemas esenciales que engloban el quehacer educativo, fundado en reflexiones que nazcan de diálogos al interior de los escenarios educativos, de manera de llegar a un estado de acción pedagógica consciente.
\end{abstract}

Palabras clave: Colaboración, diálogo, educación, saberes pedagógicos, reflexión pedagógica.

\begin{abstract}
The purpose of this reflective essay is to reflect on the processes involved in pedagogical action and supports the thesis that the emergence of the necessary pedagogical knowledge for the 21st century will be fostered by dialogicalparticipatory interactions, reflective-collaborative and situated practices carried out by teachers within their own educational contexts. The processes linked to the pedagogical field must make an effort to advance towards a reforming proposal, which invites to build a path towards transformation. Therefore, teachers must be able to understand, apprehend and think about the essential dilemmas that encompass the educational work, based on discussions that result from dialogues within the educational scenarios, in order to reach a state of conscious pedagogical action.
\end{abstract}

Key words: Collaboration, dialogue, education, pedagogical knowledge, pedagogical reflection.

\footnotetext{
${ }^{1}$ Candidata a Doctora en Educación. Universidad del Bío Bío, San Carlos, Chile. https://orcid.org/0000-0003-2777-9926 | ezagalvalenzuela@gmail.com
} 


\section{INTRODUCCIÓN}

El camino para transformarse en docente, reflexiona Contreras (2011), se encuentra lleno de paradojas, contradicciones e im/posibilidades. Todos los espacios en los que se desarrollan procesos formativos pueden ser lugares heterogéneos, aun así, ofrecen la oportunidad para la exploración y desarrollo de saberes experienciales. La complejidad, al asumir esta tarea educativa indica Olson (1995), estriba en cómo lograr que lo que se enseña cumpla una función formativa.

De este modo, la amplitud que plantea el saber pedagógico como categoría de análisis, provee diversas formas de comprensión del fenómeno educativo y el mismo arte de enseñar (Marland,1985, De Tezanos, 2007). Así pues, su acción está referida a la reflexión sobre la formación del sujeto, en la medida que este saber orienta sus acciones a unas finalidades específicas, acordes con las necesidades sociales. Como agentes del saber pedagógico, el profesorado, refiere Gadamer (1997), interpreta en cada acción, decisión y juicio, el saber pedagógico; para el autor, este sería un saber práctico que incluye el proceso de formación personal e histórica y se refleja al momento de aplicar su práctica pedagógica. Son los docentes quienes finalmente determinan y ponen énfasis en qué es enseñable y en qué momento hacerlo (Herrera \& Martínez, 2018).

Chacón \& Suárez (2006) en relación con el saber pedagógico, enfatizan que este consiste en la reconstrucción que hace el docente de sus experiencias formativas a través de cuestionamientos sobre el proceso formativo, dando lugar a la teorización, principio de la teoría pedagógica. En este sentido, Vasco et al. (2008) afirman que la teoría pedagógica, es la concreción consciente del maestro sobre sus propias prácticas formativas, desde la praxis reflexionada que hace, generalmente motivado por problemas en torno a la enseñanza, el desarrollo del currículo y la gestión educativa. Así, tanto para los procesos educativos como para la sociedad en general el profesorado asume un papel trascendental, en la medida que es este quien debe ir a la par con las necesidades que imponen las demandas sociales (Organización de las Naciones Unidas para la Educación, la Ciencia y la Cultura [UNESCO], 2014).

Feiman (2001), manifiesta que, luego de décadas de reformas y normativas educativas, se llegó al consenso de que la calidad de las instituciones educativas depende de las buenas prácticas pedagógicas del profesorado. De esta forma, los docentes son reconocidos como los actores determinantes de la acción educativa (Darling, 2010), especialmente en contextos educativos desaventajados (Organización para la Cooperación y Desarrollo Económicos [OCDE], 2018). Por tanto, fomentar políticas dirigidas hacia los docentes ha sido un componente central de las reformas desarrolladas por los países, principalmente de América Latina (Gajardo, 2020).

Especial relevancia presenta el caso chileno, ya que, a pesar de los esfuerzos realizados en orden de políticas públicas, las organizaciones escolares siguen mostrando bajos indicadores de desempeño académico (Agencia de la Calidad de la Educación, 2018, OCDE, 2014) y altos niveles de segregación escolar 
(OCDE, 2004, Valenzuela et al., 2013). Por esta razón, el presente ensayo tiene como objetivo reflexionar acerca de los procesos implicados en la acción pedagógica y sostiene la tesis de que la emergencia de los saberes pedagógicos necesarios para el siglo XXI, será propiciada, a partir de interacciones dialógicasparticipativas, prácticas reflexivas, colaborativas y situadas que realice el profesorado dentro de sus propios contextos educativos.

\section{PASADO Y PRESENTE DEL SABER PEDAGÓGICO}

El debate en torno a la teoría pedagógica, tiene larga data; sin embargo, González (2017) constató que, durante el siglo XX, varios autores plantean preguntas relativas a la práctica pedagógica y a la conformación de saberes escolares, como: “¿Qué se enseña en la escuela? ¿Por qué ciertos saberes se convierten en saberes a enseñar? (...) ¿de qué manera ciertos saberes se convierten en saberes enseñables?" (p. 46). Frente a estas interrogantes expone la autora, el proceso que da como resultado la conformación de saberes a enseñar y enseñables tiene diversas respuestas, pero la más reconocida se sintetiza en el concepto que propone Chevallard (2000), transposición didáctica. Según esta concepción, el saber científico o académico, es transformado con el fin de hacerlo plausible de ser enseñado.

En este sentido, surgen reflexiones sobre esta transposición de conocimientos al aula. Los agentes responsables de realizar los diseños curriculares y encargados de que las adaptaciones de los saberes provenientes de las producciones académicas logren decantar en las instituciones escolares, lo harían en un espacio que González (2017) denomina noosfera, escenario que se encontraría alejado de la realidad áulica. Para Bernstein (2001), este proceso transformativo ocurre, debido a la necesidad de transferir el saber desde el contexto que se produce hasta el contexto pedagógico, en el que es diseminado y reproducido. Desde esta perspectiva, la institución escolar se transforma en un espacio de reproducción del conocimiento; por tanto, el profesorado solo es un intermediario y reproductor de ese saber (González, 2017).

Álvarez (2015), por su lado postula que existe una cultura escolar con sus fronteras demarcadas, es decir, clausurada pedagógicamente. Esto significa que la escuela no constituiría un espacio en que se reproduzcan prácticas sociales y culturales, tal como lo sostuvieron autores como Bernstein (2001), Bourdieu (1997) y Giroux (2004). Por tanto, es contraria a la definición que plantea Chervel (1992), quién discute a los autores mencionados la noción de la escuela como un espacio de reproducción y se propone concebirla como un lugar de producción de una cultura. El autor argumenta su posición, afirmando que el saber escolar tiene una identidad propia, pues es una "creación histórica de la escuela" (Chervel, 1991, p. $65)$.

En relación con la cultura escolar, Julia (2001), la considera como "un conjunto de normas que definen conocimientos a enseñar y conductas a inculcar, y un conjunto de prácticas que permiten la transmisión de esos conocimientos y la incorporación de esos comportamientos" (p.10). La historia de la cultura escolar ha 
experimentado importantes avances en los que se destacan estudios provenientes de México, Colombia, España y el mundo anglosajón, y más recientemente en Brasil y Argentina (Álvarez, 2015). Se destacan en este aspecto autores como Cuesta (2001), Viñao (2012), Escolano (2006), quienes han profundizado la temática desde España.

Viñao (2012), retoma en sus argumentos la cultura escolar y disciplinar escolar e indica que las disciplinas escolares son construcciones, productos históricos que se configuraron y modificaron en el transcurso del tiempo en y por la cultura escolar. En este sentido, quien contribuyó a consolidar la línea histórica de las disciplinas escolares en América Latina fue Goodson (1991). Para el autor, el currículo escolar no es un producto técnico, racional, imparcial y sintetizador del conocimiento académico, sino resultado de diversas luchas sociales y políticas.

Entonces, se podría señalar que el saber pedagógico es un discurso que se establece no solo por la recopilación de otros saberes, sino a partir de las prácticas de formación e instrucción de estos. Es decir, que los conocimientos disciplinarios se transforman en objetos de enseñanza al ser procesados didácticamente, sistematizados y registrados (Restrepo, 2004). Runge (2002), propone que se compone de variados discursos relativos a la enseñanza en el sentido teórico o práctico, reunidos en temáticas como: educación, instrucción, pedagogía y enseñanza.

Lo anterior remite a una concepción propuesta por Foucault (1991) respecto de la configuración del saber, que para el autor se encontraría vinculada a la "práctica discursiva". Para este filósofo, el saber se conforma a partir de los elementos y formas de estar en una práctica a la que solo se puede acceder mediante el discurso. A lo que Zuluaga (1999) agrega:

El discurso del saber pedagógico se encuentra disperso en diversos registros que recogen (información de:) a) Los conceptos propios de la historicidad de la pedagogía [...] b) La difusión llevada a cabo por los organismos del Estado [...] c) La adecuación social a que es sometido el discurso para efectos del poder político y económico [...] d) La producción de saber por los sujetos de saber que delimita para las instituciones de la práctica pedagógica el proceso de institucionalización [...] e) Las adecuaciones que producen en el saber pedagógico otros saberes hegemónicos [...] f) Los registros propios de las instituciones donde se realizan prácticas pedagógicas [...] g) Las normas que provienen del propio saber [...] h) Las posiciones de sujetos de saber que puede asumir un maestro en nuestra formación social. (p.40)

Respecto del debate pedagógico en torno a la educación, históricamente ha transitado dentro de disputas potenciadas por intereses políticos y económicos, que han condicionado las maneras en que se movilizan los sujetos en el mundo. Se ha supeditado el modo de ser y estar, además de irrumpir en las formas de pensar la educación del siglo XXI, y en consecuencia en el ejercicio pedagógico. El surgimiento de diversas metodologías educativas, basadas en tradiciones como la alemana, francesa, anglosajona y americana, con 
sus respectivas propuestas influyeron directamente en la emergencia de los saberes pedagógicos. Por tanto, se comprende, a la luz del propósito de este escrito, el saber pedagógico como una interpretación discursiva del mundo, así pues, toda práctica pedagógica como la materialización interpretativa de dichos discursos.

Con todo, la construcción de los procesos pedagógicos continúa replicando el modelo fabril o fordista, propio del capitalismo cognitivo en el que, "los procesos industriales exigen la observancia de unas normas y unos niveles de calidad específicos. Este principio (capitalista) aún se aplica en educación" (Robinson, 2016, p. 69). Con ello, el saber pedagógico en la actualidad se produce bajo una concepción técnicoinstrumental, mediada por criterios administrativos de eficiencia y eficacia (Hernández, 2020, Muñoz \& Rodríguez, 2017, Pérez, 2016), criterios que se evalúan bajo códigos que responden a distinciones binarias (mejor/peor, bueno/malo, apto/no apto, aprobó/no aprobó). Por esta razón, no se debe olvidar lo expuesto por Delors (1996):

Una nueva concepción más amplia de la educación debería llevar a cada persona a descubrir, despertar e incrementar sus posibilidades creativas, actualizando así el tesoro escondido en cada uno de nosotros, lo cual supone trascender una visión puramente instrumental de la educación. (p. 96).

Lo expuesto induce a una mirada retrospectiva, acerca de la esencia, valores y fines de la educación. En este sentido, es valedero cuestionar el futuro de la pedagogía, la que hasta el momento mantiene el statu quo y prioriza enfoques globales. De momento, contextos territoriales, acervo de conocimientos o miradas inductivas sobre los procesos pedagógicos que consideren los saberes comunitarios y participativos, son deslegitimados y cubiertos con sedimentos de la normativa curricular centralizada.

Por lo descrito en los acápites anteriores, surge la necesidad como afirma, Zuluaga et al. (2014) de una reconceptualización de la pedagogía y pensarla desde el pensamiento filosófico; ser consientes desde dónde se está desarrollando los saberes pedagógicos, desde qué fundamentación se está practicando la pedagogía y para qué lo hacemos. De lo contrario, se continúa abonando la tecnificación del saber pedagógico, olvidando la conciencia pedagógica, y así, el ejercicio de pensar en una educación, que considere, en palabras de Morin (1999), los saberes necesarios para la educación del futuro, con la disposición de contemplar, más allá de lo disciplinar, la adquisición de saberes emocionales, inclusivos, pluriculturales, artísticos, ambientales, patrimoniales, sexoafectivos, comunicacionales, relativos a la equidad de género, por mencionar algunos ejemplos. 


\section{DESDE LOS INICIOS DE LAS PRÁCTICAS PEDAGÓGICAS EN CHILE A LA EMERGENCIA DEL SABER PEDAGÓGICO ACTUAL}

Los primeros modelos educativos puestos en funcionamiento en Chile, tuvieron influencias tanto de la cristianización como de doctrinas políticas europeas, ideales que se intentaban transmitir a la población y que eran herencia de la llegada de españoles y portugueses a Sudamérica, quienes en su proceso de conquista traían aparejada la fe católica. Durante el período de la colonia (siglos XVI-XVIII), la educación que predominó en Chile, fue la vinculada a las congregaciones religiosas (mercedarios, 1535; franciscanos, 1553; dominicos, 1557; jesuitas, 1593 y agustinos 1595) los que promovían la incorporación de las primeras letras y que, como preludio, su intención era adoctrinar a la población para que pudiesen efectuar los rezos cristianos (Caiceo, 2009).

Frontaura (1892), en un esfuerzo por reconstruir históricamente los cimientos de la educación pública en Chile redacta el texto, Noticias históricas sobre las escuelas públicas de chile a fines de la era colonial publicado en 1892. En su estudio, da a conocer la estructura y los métodos utilizados en la educación chilena, durante el período colonial. Sostiene que los estudiantes, por medio de Las Cartillas, comenzaban con el proceso de lectoescritura; leían Cristo, á, bé, ce y continuaban hasta conocer todas las letras. El autor relata la exigencia de este proceso, de la siguiente manera:

Los muchachos deletreaban en voz alta, á gritos, todos á un tiempo. Desde la puerta de la escuela no se oía á ciertas horas otra cosa que un gran murmullo, una inmensa algazara y una voz más alta que los demás que gritaba: be, á; ba: be, e, be: be, i; bi: be, ó; bo: be, ú; bu: etc. [...] Después de deletrear, iban los niños lentamente ejercitándose en juntar las sílabas, hasta formar palabras, otro escollo que algunos sólo pasaban á fuerza de latigazos y después de largos meses de ejercicio. (p.33).

Posteriormente, se incorporaron los preceptores a la labor educativa, quienes se desempeñaban en las aulas públicas de instrucción primaria, cuya la labor fundamental era lograr que los niños y niñas pudiesen leer, escribir, contar y los principios básicos de la doctrina cristiana. Recuerda la metodología utilizada por estos preceptores en los inicios republicanos de Chile, Pérez Rosales (1886), quien deja registro descriptivo de estas prácticas pedagógicas, en su obra Recuerdos del pasado 1814-1860.

El profesor o dómine, quien, como todos los de su especie entonces, podia llamarse don Tremendo, ocupando en alto una de las cabecera del salón, ostentaba sobre la mesa que tenía por delante, al lado de algunas muestras de escritura i de tal cual garabateando Caton, una morruda palmeta con sus correspondiente látigo, verdaderos propulsores de la instrucción i del saber humano en una época en que se encontraba sumo chiste i mucha verdad al dicho brutal: la letra con sangre entra. (p. 9). 
Este preceptor para Pérez Rosales, además de ser el poseedor máximo del saber humano, entregaba dichos conocimientos de forma autoritaria y violenta. Más que huellas académicas en sus estudiantes, dejaba marcas que lo hacían merecedor de una categoría, especie tremenda, tal como lo describe el autor. Queda de manifiesto, por tanto, que en los inicios de los procesos de escolarización en el país predominó una práctica pedagógica con una estructura rígida. Esto conlleva, como afirma Hernández (2020), a un saber basado en el "reduccionismo cientificista y que por ello requiere ser repensado y construido desde la pasión creadora" (p.8).

Ciertamente, desde estos relatos transcurrieron casi dos siglos y la construcción del saber pedagógico en el contexto chileno evolucionó y se complejizó en la medida que fue alcanzando un mayor nivel de especialización; aunque siempre influenciado por los hitos históricos que ha vivenciado el país y por posturas metodológicas provenientes del exterior, asimismo, resultantes de otros contextos tanto sociales como políticos.

El saber pedagógico, ligado estrechamente con el ejercicio de la profesión docente, exige al profesorado la constantemente reconstrucción de su actuar. Los espacios cuadrangulares de las salas de clases ya no permiten abarcar todo lo que se puede llegar a enseñar y aprender, de la forma en que se concebía en épocas anteriores. Por el contrario, se convirtió en un espacio en el que se contiene a los estudiantes para que pasen más de doce años de sus vidas aprendiendo contenidos que en su mayoría podrían aprender de manera autodidacta (Lisman, 2021). Lo anterior, se reflejó aún más durante la actual pandemia del SARS-CoV-2, donde el aula se volvió híbrida y virtual.

De esta manera, la educación como fenómeno social implica la transformación cognitiva e histórica de quienes habitan los territorios. A partir de esta premisa surge la discusión en torno a la valoración del papel profesional del profesorado (Fandiño \& Bermúdez, 2015). Al respecto, se han desarrollado estudios orientados hacia el valor particular del saber pedagógico, en su estrecha vinculación con la práctica educativa; trabajos como los de Chacón \& Suárez (2006), De Tezanos (2007), Mercado (2002), Restrepo (2004), Runge (2002), Tardif (2014), Vasco et al. (2008) y Zuluaga (1999), fundamentan el saber pedagógico como un saber construido a partir de la experiencia del ejercicio de la profesión, abarcando además la acción reflexiva que se genera sobre la práctica pedagógica. Según Barrera (2009), a partir del saber que se construye producto de la experiencia del ejercicio de la profesión, como por el que se genera a través de la acción reflexiva alrededor de la práctica, la particularidad del saber de la enseñanza impone la coherencia de toda política de desarrollo del profesorado. Situación, afirma el autor mencionado, "equivalente en el reconocimiento de su saber, como de las condiciones necesarias para su progreso" (Barrera, 2009, p. 42).

Los estudios de Álvarez et al. (2011) dan cuenta que lo aplicado en la práctica pedagógica está impregnado de la apropiación de conocimiento que ha adquirido el profesorado a lo largo de la vida y trayectoria académica. De esta manera, se espera que la atención por parte de las investigaciones se centre 
en la emergencia de los saberes pedagógicos situados, es decir, profundizar en las acciones pedagógicas que emergen de situaciones locales, que nacen en las propias comunidades y de las reflexiones que se originan al interior de estas y que se realizan mediante el diálogo comunitario.

Como plantea Haraway (1991), se debe asumir el conocimiento situado, encarnado, desde una mirada parcial, desde la posición en la que me encuentro y el contexto donde me sitúo; es decir, desde las parcialidades donde nace el conocimiento y no desde la universalidad. Todo conocimiento se produce en situaciones históricas y sociales particulares, como se ha insistido en este escrito. En este sentido, la postura propuesta por Díaz (2006), entrega herramientas para justificar la construcción de saberes relativos al quehacer pedagógico dentro de ambientes reales de aprendizaje y contextos socioculturales con características análogas. Lo mencionado, como elemento clave para ocuparse de situaciones cotidianas, mediante el apoyo de la colectividad, por cuanto lo que se está viviendo, construyendo y reproduciendo se origina bajo experiencias similares.

\section{HACIA EL ENCUENTRO DEL OTRO: COMUNIDADES COLABORATIVAS PARA EL PROGRESO DE LA LABOR PEDAGÓGICA}

Uno de los aspectos que considera la Evaluación Docente ${ }^{1}$ que realiza el profesorado en Chile refiere al trabajo colaborativo que realizan los docentes en su quehacer cotidiano. En dicha tarea deben dar cuenta de experiencias de trabajo en conjunto en las que hayan tenido la oportunidad de dialogar y reflexionar en torno a aspectos pedagógicos, ya sea con otros docentes, educadoras, profesionales o asistentes de la educación, integrantes de la comunidad educativa, docentes del microcentro o de redes en las cuales participan (DocenteMÁS, 2021). Se debe mencionar que este apartado (trabajo colaborativo) no es obligatorio dentro del portafolio docente.

Los resultados entregados por el Ministerio de Educación de Chile (Mineduc, 2020) sobre el proceso de evaluación docente 2019, reveló que, de las $17.244^{2}$ evaluaciones realizadas por los docentes, un $66 \%$ de quienes realizaron el portafolio presentó evidencias del trabajo colaborativo. Los resultados obtenidos e indicadores considerados, se describen en la Tabla 1.

\footnotetext{
1 El Sistema de Evaluación del Desempeño Profesional Docente es una evaluación obligatoria para los docentes, educadoras y educadores de aula que se desempeñan en establecimientos que dependen de los municipios o de los Servicios Locales de Educación y voluntaria para docentes de establecimientos particulares subvencionados a lo largo del país. Su objetivo es fortalecer la profesión docente y contribuir a mejorar la calidad de la educación (DocenteMÁs, 2021).

2 El número de evaluaciones, 17.244 corresponde solo a evaluaciones de docentes que se desempeñan en dependencias municipales o de los Servicios Locales de Educación, ya que el total de evaluaciones realizadas durante el año 2019 corresponde a 21.097.
} 
Tabla 1

Resultados por aspecto evaluado en el Portafolio: Trabajo Colaborativo.

\begin{tabular}{lc}
\multicolumn{1}{c}{ Indicadores } & Porcentaje alcanzado \\
\hline $\begin{array}{l}\text { Relevancia de la necesidad que motivó } \\
\text { el trabajo colaborativo. }\end{array}$ & $33.9 \%$ \\
\hline Calidad del diálogo profesional. & $23.3 \%$ \\
\hline $\begin{array}{l}\text { Valor del trabajo colaborativo para el } \\
\text { desarrollo profesional. }\end{array}$ & $14.3 \%$ \\
\hline $\begin{array}{l}\text { Reflexión sobre el impacto de la } \\
\text { experiencia de trabajo colaborativo. }\end{array}$ & $18.8 \%$ \\
\hline $\begin{array}{l}\text { Relevancia de la necesidad que motivó } \\
\text { el trabajo colaborativo. }\end{array}$ & $33.9 \%$ \\
\hline
\end{tabular}

Fuente: Elaborado a partir de (Mineduc, 2020).

Como se observa, los resultados develan la imperiosa necesidad de incentivar el trabajo colaborativo. Visto que, la motivación, calidad del diálogo, valoración o reflexión que realizan los docentes sobre su desempeño en esta área, reflejan grandes debilidades. En consecuencia, generar propuestas o intervenciones educativas que fomenten comunidades participativas dentro de las organizaciones escolares, no sería una prioridad del profesorado. Las razones que tendrían los docentes para alejarse de estas prácticas no estarían relacionadas directamente con la falta de voluntad profesional, sino más bien con factores tanto políticos como administrativos relativos al sistema educativo.

Ávalos \& Valenzuela (2016), en la investigación que realizaron por medio del Centro de Investigaciones Avanzada en Educación de la Universidad de Chile (CIAE) reveló que cerca de 40\% de docentes noveles abandonan el sistema escolar, situación, sostienen los autores, que es motivada por las malas condiciones laborales y el agotamiento emocional. De esta manera, la situación laboral del profesorado también podría impactar en los vínculos que se establecen al interior de las organizaciones educativas impidiendo el desarrollo de prácticas colaborativas.

Respecto de promoción de acciones dialógicas participativas de los docentes al interior de los establecimientos educacionales, se han desarrollado investigaciones como las de: Arévalo \& Núñez (2016), Vaillant (2016), Krichesky \& Murillo (2011). En dichos estudios se consideran las prácticas colaborativas como una estrategia que logra abrir espacios para el rescate, construcción y reconstrucción de saberes pedagógicos acumulados. Así pues, por medio de la interacción entre docentes noveles y experimentados, se establecerían puentes y nexos hacia una acción comunicativa, en la que se logre reflexionar, pensar, cuestionar y desarrollar prácticas pedagógicas efectivas. Para Habermas (1999) la acción comunicativa es: 
[...] la interacción de al menos dos sujetos capaces de lenguaje y de acción que (ya sea con medios verbales o con medios extraverbales) entablan una relación interpersonal. Los actores buscan entenderse sobre una situación de acción para poder así coordinar de común acuerdo sus planes de acción y con ello sus acciones (p.124).

Las reflexiones que realiza Habermas no refieren precisamente a la pedagogía, más bien tratan de explicar el orden social; sin embargo, establecen una línea reflexiva para una teoría de la educación (García, 1993). Precisamente, esta línea, es la que genera el interés de este escrito, puesto que se piensa que, para lograr procesos transformativos en educación, se debe comenzar a discutir sobre las prácticas metacomunicativas que nacen de las dinámicas de interacción comunicativa de los actores del sistema escolar, específicamente los vínculos que se generan para la acción pedagógica entre el profesorado.

De esta manera, las interacciones comunicacionales que se podrían producir en los espacios donde se construyen saberes pedagógicos, se estructuran alrededor de la interacción entre los participantes (docentes), los cuales deben estar en condición de distinguir lo que se percibe colectivamente e ir mucho más allá de un diálogo direccional entre un emisor y receptor. Por lo que las selecciones de sentido que operan en las comunicaciones deben realizarse tomando en cuenta a todos los participantes (Urquiza et al., 2018).

Las instancias dialógicas-colaborativas no emergen espontáneamente, por ello las reuniones o consejos de profesores $/ \mathrm{as}^{3}$, más allá de tratar temas administrativos, deben transformarse en espacios de verdadera reflexión pedagógica y realizarse en lo que se podría denominar diálogos de mesa redonda con temáticas pedagógicas que no emerjan espontáneamente, sino que tengan una dimensión teleológica, considerando los fenómenos complejos de su realidad y desarrolladas transdisciplinariamente (Cruz, 2011). Todo proceso dialógico no se encuentra exento de dificultades o conflictos comunicacionales, producto muchas veces del establecimiento de jerarquías, las que finalmente solo consiguen alejar de la comunicación a los interlocutores. Por ello, el desafío consiste en superar estas dificultades mediante la propuesta dialógicacolaborativa, puesto que la acción pedagógica no es individual sino colectiva.

\footnotetext{
${ }^{3}$ Los Consejos de profesores/as dentro de las organizaciones escolares son organismos técnicos de carácter consultivo, conformados por docentes directivos, técnico-pedagógicos y docentes en general, donde cada uno de sus integrantes expresa su opinión y reflexiones profesionales según la Ley Orgánica 20.501/2011, de 8 de febrero para la Calidad y Equidad de la Educación. https://www.ilo.org/dyn/natlex/docs/ELECTRONIC/86201/97129/F537355554/CHL86201.pdf
} 


\section{CONCLUSIONES}

La exposición reflexiva presente en este ensayo se traza desde la convicción que para el desarrollo de saberes pedagógicos propicios para el siglo XXI, estos deben asentarse sobre una propuesta reformadora, que invite a construir un camino hacia la transformación educativa. Así como, tan lucidamente lo destacó Morin (2011) “Porque solo la metamorfosis podría mejorar el mundo" (p.283).

Para que esta transformación logre ver la luz es necesario ser capaz de comprender, aprehender y reflexionar acerca de los dilemas esenciales que engloban el quehacer educativo. Resulta imperante establecer diálogos-participativos en espacios de colaboración que tributen a la generación de saberes pedagógicos fundados en el bien-estar, bien-ser, bien-actuar, basados en la profunda sabiduría ancestral como es el buen vivir. Una suerte de diálogo entre el conocimiento científico y conocimiento situado, de modo que se encarne en lo educativo.

Finalmente, para la emergencia de saberes pedagógicos situados, estos necesitan ser cultivados en escenarios educativos donde se promueva la conciencia pedagógica por medio de la reflexión en torno a problemáticas y aciertos observados durante el proceso de acción pedagógica. Lo anterior no fundado desde la interacción con matices individualistas, sino más bien desde una disposición a la colaboración como un sistema, como un equipo con un objetivo en común, que es educar.

\section{REFERENCIAS BIBLIOGRÁFICAS}

Agencia de la Calidad de la Educación. (2018). Informe Nacional de la Calidad de la Educación 2017. Los desafíos de educar para la inclusión y la diversidad. https://archivos.agenciaeducacion.cl/IDH_web.pdf

Álvarez, F., Abrahams, M., Gaete, C., Galdames, V., Latorre, M., Lee M., \& Rojas, M. (2011). Saber pedagógico y formación inicial de docentes. Facultad de Educación. Universidad Alberto Hurtado.

Álvarez-Álvarez, C. (2015). Teoría frente a práctica educativa: Algunos problemas y propuestas de solución. Perfiles Educativos, 37(148), 172-190. https://doi.org/10.1016/j.pe.2015.11.014

Arévalo, A., \& Núñez, M. (2016). Buscando comprender la dimensión de lo colaborativo. Los profesores hablan. Docencia, (60), 55-65. https://www.docentemas.cl/portafolio/wpcontent/uploads/2018/05/Buscando-comprender-la-dimensi\%C3\%B3n-de-lo-colaborativo.pdf

Ávalos, B., \& Valenzuela J.P. (2016). Education for all and attririon/retention of new teachers: A trajectory study in Chile. International Journal of Education Development, 49, 279-290. https://doi.org/10.1016/j.ijedudev.2016.03.012 
Barrera, F. (2009). Desarrollo del profesorado: el saber pedagógico y la tradición del profesor como $\begin{array}{lllll}\text { profesional } & \text { reflexivo. } & \text { Acción } & \text { Pedagógica, }\end{array}$ http://www.revencyt.ula.ve/storage/repo/ArchivoDocumento/accion/v18n1/articulo4.pdf

Bernstein, B. (2001). La estructura del discurso pedagógico. Clases, códigos y control. Morata.

Bourdieu, P. (1997). Razones prácticas. Sobre la teoría de la acción. Anagrama.

Caiceo, J. (2009). Estado, iglesia y sistema educativo durante la república en Chile. Revista HISTEDBR, 9(35), 3-18. https://doi.org/10.20396/rho.v9i35.8639610

Chacón, M., \& Suárez, M. (2006). La valoración del saber pedagógico desde la relación teoría-práctica: Una consideración necesaria en la formación de docentes. Universitas Tarraconensis: Revista de ciències de l'educació, (1), 301-311.

Chervel, A. (1992). L'école, lieu de production d'une culture. En F. Audigier \& G. Baillat (Eds.), Didactique de l'histoire, de la géographie, des sciences sociales. Analyser et gérer les situations d'enseignementapprentissage, (195-198). INRP.

Chervel, A. (1991). Historia de las disciplinas escolares: Reflexiones sobre un campo de investigación. Revista de Educación, 295, 59-111. https://redined.mecd.gob.es/xmlui/handle/11162/70295

Chevallard, I. (2000). La transposición didáctica. Del Saber Sabio Al Saber Enseñado.AIQUE. https://nelsonreyes.com.br/LIVRO_LA\%20TRANSPOSICION\%20DIDACTICA.pdf

Contreras, D. (2011). El lugar de la experiencia. Cuadernos de pedagogía, (417), 60-63. https://redined.educacion.gob.es/xmlui/handle/11162/37579

Cruz, L. F. (2011). Educación siglo XXI y sistemas complejos. http://sedici.unlp.edu.ar/handle/10915/72664

Cuesta, R. (2001). Voces y ecos de la enseñanza de la historia en España (1875-1936). Aula, 13, 79-93. https://redined.educacion.gob.es/xmlui/handle/11162/208769

Darling-Hammond, L. (2010). Teacher education and the American future. Journal of Teacher Education 61(1-2), 35-47. https://doi.org/10.1177/0022487109348024

De Tezanos, A. (2007). Oficio de enseñar- saber pedagógico: La relación fundante. Educación y Ciudad, 12, 7-26. https://doi.org/10.36737/01230425.n12.175

Delors, J. (1996). La educación encierra un tesoro. Informe a la UNESCO de la Comisión internacional sobre la educación para el siglo XXI. Madrid. Santillana/UNESCO.

Díaz Barriga, F. (2006). Enseñanza Situada, (2ª Ed.). McGraw Hill.

DocenteMÁS (2021). Sistema de Evaluación del Desempeño Profesional Docente. Ministerio de Educación de Chile. https://www.docentemas.cl/ 
Escolano, B. (2006). Historia ilustrada de la escuela en España: dos siglos de perspectiva histórica. Historia de la Educación, 25, 651-652. https://revistas.usal.es/index.php/02120267/article/view/11209/11630

Fandiño, Y., \& Bermúdez, J. (2015). Práctica pedagógica: subjetivar, problematizar y transformar el quehacer docente. En: R. Páez (Ed.), Práctica y experiencia: claves del saber pedagógico docente (pp. 29-54). Unisalle.

Feiman-Nemser, S. (2001). Helping novices learn to teach lessons from an exemplary support teacher. Journal of Teacher Education. 52(1), 17-30. https://doi.org/10.1177/0022487101052001003

Foucault, M. (1991). Microfísica del poder. La piqueta.

Frontaura, J. (1892). Noticias históricas sobre las escuelas públicas de Chile a fines de la era colonial: su origen, número, maestros, alumnos, castigos, textos, métodos de enseñanza, é importancia que ellos tuvieron en el éxito de la revolución de independencia. Imprenta Nacional. http://www.memoriachilena.gob.cl/602/w3-article-62098.html

Gadamer, H. (1997). Verdad y método I. Sígueme.

Gajardo, M. (2020). Desarrollo educativo en América Latina. Tendencias globales. Desafíos Regionales. FLACSO-Chile. https://flacsochile.org/doc/2020/Desarrollo_Educativo_en_AL_Nov_2020.pdf

García, J. (1993). Acción pedagógica y acción comunicativa. Reflexiones a partir de textos de J. Habermas. Revista de Educación, (302), 129-164. https://www.educacionyfp.gob.es/dam/jcr:d7a46588e364-4440-a052-a11ab77f631e/re3020700490-pdf.pdf

Giroux, H. (2004). Cultural Studies, Public Pedagogy, and the Responsibility of Intellectuals. Communication and Critical/cultural Studies, 1(1), 59-79. https://doi.org/10.1080/1479142042000180926

González, M. (2017). Los saberes históricos escolares como construcción situada y singular. Diálogo andino, 53, 45-57. http://dx.doi.org/10.4067/S0719-26812017000200045

Goodson, I. (1991). La construcción social del currículum. Posibilidades y ámbitos de investigación de la $\begin{array}{lllll}\text { Historia del } & \text { currículo. Revista de }\end{array}$ https://www.educacionyfp.gob.es/dam/jcr:6d91bb79-2ab0-419b-945ea68624727d35/re29501-pdf.pdf

Haraway, D. (1991). Ciencia, cyborgs y mujeres: La reinvención de la naturaleza. Cátedra.

Habermas, J. (1999). Teoría de la acción comunicativa, I. Racionalidad de la acción y racionalización social. Taurus.

Hernández, J. (2020). El saber pedagógico anarquista: Una interpretación desde la hermenéutica analógica. [Tesis de maestría]. Universidad Nacional Autónoma de México. 
Herrera, J., \& Martínez, Á. (2018). El saber pedagógico como saber práctico. Pedagogía y Saberes, (49), 9-26. http://www.scielo.org.co/pdf/ppo/n19/2011-804X-ppo-19-00009.pdf

Julia, D. (2001). A cultura escolar como objeto histórico. Revista brasileira de história da educação. 1 (1 [1]), 9-43. https://periodicos.uem.br/ojs/index.php/rbhe/article/view/38749

Krichesky, G., \& Murillo, J. (2011). Las comunidades profesionales de aprendizaje. Una estrategia de mejora para una concepción de escuela. Revista Iberoamericana sobre Calidad, Eficacia y Cambio en Educación, 9(1), 65-83. https://www.redalyc.org/pdf/551/55118790005.pdf

Lisman, A. (2021). Pedagogía incendiaria. La escuela tiene los días contados. Descontrol.

Marland, M. (1985). El arte de enseñar: técnicas y organización del aula. Morata.

Mercado, R. (2002). Los saberes docentes como construcción social. La enseñanza centrada en los niños. Fondo de Cultura Económica.

Ministerio de Educación de Chile [Mineduc]. (2020). Informe Resultados Nacionales Evaluación Nacional Diagnóstica de la Formación Inicial Docente 2019. Centro de Perfeccionamiento, Experimentación e Investigación- CPEIP. Ministerio de Educación. https://www.cpeip.cl/wpcontent/uploads/2020/08/Informe-Nacional-END-2019_rect.pdf

Morin, E. (1999). Los siete saberes necesarios para la educación del futuro. UNESCO.

Morin, E. (2011). Para el futuro de la humanidad. PAIDÓS.

Muñoz, M., \& Rodríguez, E. (2017). Priorización de lo técnico-instrumental sobre la filosofía en la formación de docentes para el siglo XXI. 4to Congreso Latinoamericano de Filosofía de la Educación. Universidad Nacional de San Martín, Buenos Aires, Argentina.

Organización de las Naciones Unidas para la Educación, la Ciencia y la Cultura [UNESCO]. (2014). Informe de Seguimiento de la Educación para Todos 2013/4. http://unesdoc.unesco.org/ images/0022/002261/226159s.pdf

Organización para la Cooperación y Desarrollo Económicos [OCDE]. (2018). Effective Teacher Policies, Insights from PISA. PISA OECD Publishing. https://doi.org/10.1787/9789264301603-en

Organización para la Cooperación y Desarrollo Económicos [OCDE]. (2014). TALIS 2013 Results: An International Perspective on Teaching and Learning. OECD Publishing. https://doi.org/10.1787/9789264196261-en

Organización para la Cooperación y Desarrollo Económicos [OCDE]. (2004). Revisión de políticas nacionales de educación: Chile. París: Organization for Economic Co-operation and Development (OCDE). https://bibliotecadigital.mineduc.cl/handle/20.500.12365/17435 
Olson, M. (1995). Conceptualising narrative authority: implication for teacher education. Teaching and Teacher Education, 11(2), 119-135. https://doi.org/10.1016/0742-051X(94)00022-X

Pérez Rosales, V. (1886). Recuerdos del pasado, 1814-1860. Zig-zag.

Pérez, J. (2016). Reciprocidad socio-pedagógica del educando ante la praxis técnico-instrumental del docente. Saber, 28(4), 838-850. http://ve.scielo.org/pdf/saber/v28n4/art19.pdf

Restrepo, B. (2004). La investigación-acción educativa y la construcción de saber pedagógico. Educación y Educadores, 7, 45-55. https://www.redalyc.org/pdf/834/83400706.pdf

Robinson, K. (2016). Escuelas Creativas. La revolución que está transformando la educación. Grijalbo.

Runge, A. (2002). Una epistemología histórica de la pedagogía: El trabajo de Olga Lucía Zuluaga. Revista de Pedagogía, 23(68), 361-385. $\quad$ http://ve.scielo.org/scielo.php?pid=S0798$97922002000300002 \&$ script=sci_abstract

Tardif, M. (2014). Los saberes del docente y su desarrollo profesional. Narcea.

Urquiza, A., Amigo, C., Billi, M., Brandao, G. y Morales, B. (2018). Metálogo como herramienta de colaboración $\begin{array}{llll}\text { transdisciplinaria. } \quad \text { Cinta } & \text { moebio, }\end{array}$ https://cintademoebio.uchile.cl/index.php/CDM/article/view/49461

Vaillant, D. (2016). Trabajo colaborativo y nuevos escenarios para el desarrollo profesional docente. Docencia, 16, 5-13. $\quad$ https://www.docentemas.cl/portafolio/wpcontent/uploads/2018/05/Trabajo-colaborativo-y-nuevos-escenarios-para-el-desarrolloprofesional-docente.pdf

Valenzuela, J.P., Bellei, C., \& De los Ríos, D. (2013). Socioeconomic school segregation in a market-oriented educational system: The case of Chile. Journal of Education Policy, 29(2), 217-241. https://doi.org/10.1080/02680939.2013.806995

Vasco, C., Martínez, A., \& Vasco, E. (2008). Educación, pedagogía y didáctica: una perspectiva epistemológica. En G. Hoyos (Ed.). Enciclopedia Iberoamericana de Filosofía. Filosofía de la Educación. (Vol. 29, pp. 99-127). Trotta-Consejo Superior de Investigaciones Científicas CSIC. https://www.udocz.com/apuntes/135812/educacion-pedagogia-y-didactica-una-pers

Viñao, A. (2012). Historia de las disciplinas, profesionalización docente y formación de profesores: el caso español. Pro-Posições, $\quad$ 23(3), 103-118. https://periodicos.sbu.unicamp.br/ojs/index.php/proposic/article/view/8642838

Zuluaga, O. (1999). Pedagogía e historia. Siglo del Hombre, Anthropos, Universidad de Antioquia.

Zuluaga, O., Exheverri, A., Martínez, A., Quinceno, H., Saenz, J., \& Álvarez, A. (2014). Pedagogía y Epistemología. Magisterio. 
Zagal, E. (2021). Colaboración, diálogo y reflexión: Una mirada hacia la emergencia de los saberes pedagógicos del siglo XXI. Revista Convergencia Educativa, (10), diciembre, 7-22. https://doi.org/10.29035/rce.10.7

\section{Datos de correspondencia}

\section{Evelyn Zagal Valenzuela}

Candidata a Doctora en Educación

Universidad del Bío Bío.

San Carlos, Chile.

Dirección postal: 3840000

ORCID ID: https://orcid.org/0000-0003-2777-9926

Email: ezagalvalenzuela@gmail.com 